\title{
Description of three new Pipunculus Latreille, 1802 species (Diptera: Pipunculidae) from Central Europe and Turkey
}

\author{
Milan KozÁneK ${ }^{1}$, Daniel ValašKa ${ }^{1}$, Ján Kodada ${ }^{2}$ \\ ${ }^{1}$ VirNat s.r.o., Na Medzi 1C, Bratislava, SK-831 06, Slovakia, ${ }^{2}$ Department of Zoology, Faculty of Natural Sciences, Comenius University in Bra- \\ tislava, Ilkovičova 6, Bratislava, SK-842 15, Slovakia
}

Corresponding author: Dr. Milan Kozánek, VirNat s.r.o., Na Medzi 1C, SK-831 06, Bratislava, Slovakia. E-mail: milan@scientica.sk

Recieved: 10 June 2021 Accepted: 20 June 2021 Published online:

7 December 2021

Subject editor:

Dávid Selnekovič

\begin{abstract}
Extensive sampling of Pipunculidae in the frame of faunistic research in former Czechoslovakia as well as several other European countries over the last two decades has resulted in the discovery of several unknown species of this family. In this work, we present descriptions of three new species of the genus Pipunculus Latreille, 1802. Pipunculus adami Kozánek, sp. nov. and $P$. lindae Kozánek, sp. nov. are described from Slovakia and the Czech Republic, P. anatolicus Kozánek, sp. nov. is described on the basis of specimens from Turkey. The detailed redescription of the male of $P$. wolf Kowarz, 1887, including the so far unknown male terminalia, is presented. The key taxonomic characters are visualized as interactive microphotographs provided with browsing software.
\end{abstract}

Key words: big-headed flies, taxonomy, microphotographs, visualisation, characters.

\section{Introduction}

The research of European Pipunculidae accelerated after the 1980 s when a number of works revising world, European or regional fauna of pipunculid genera were published (Kozánek 1981, De Meyer 1989, Albrecht 1990, Jervis 1992, Földvári \& De Meyer 1999). A substantial step ahead brought the works of Kehlmaier who revised European fauna of Eudorylini (Kehlmaier 2005), Chalarus Walker, 1834 (Kehlmaier \& Assmann 2008), Verrallia Mik, 1899 (Kehlmaier 2006), Jassidophaga Aczel, 1939 (Kehlmaier 2006), and Pipunculus Latreille, 1802 (Kehlmaier 2008). Our knowledge on the pipunculid distribution in European countries is not uniform. While the pipunculid fauna of Western, Northern, and Central European countries is well studied, South ern and Eastern European countries need more precise faunistic research. Two new Pipunculus from Slovakia and the Czech Republic described in present work demonstrate that continuing faunistic studies can reveal new unknown species even in countries with long tradition of Pipunculidae research.

The significant progress in the research of Pipunculidae taxonomy and phylogeography over the last decades has allowed the implementation of modern methods of molecular biology, but the description of new species and identification is still based on the visualization of key morphological characters. Drawings of these characters, mainly male and female terminalia, are still widely used. To eliminate differences in the quality of the drawings, several authors used composed microphotographs created by stacking of several focal planes (Kehlmaier et al. 2019, Motamedinia et al. 2020). In this study we use interactive microphotographs for visualization of key characters of described species. The principle of this method is based on the combination of high resolution stacked microphotography with browsing software allowing manipulation of the object.

\section{Material and methods}

The described species are the result of the study of extensive Pipunculidae material collected by Malaise trap and sweeping net from the Czech Republic, Slovakia and Turkey. The Malaise traps collecting medium was $70 \%$ ethanol denaturized with isopropyl alcohol. Collected specimens were desiccated by transferring through $98 \%$ ethanol-98\% ethanol : ethylacetate $(1: 1)$ - ethylacetate desiccating series. The type series of the described species and the studied material of $P$. wolf $i$ are deposited in the Slovak National Museum, Bratislava, Slovakia (SNM). The description of each presented species is completed by interactive microphotographs of their respective key characters. In order to create stacked microphotographs, a macroscope Zeiss Axio Zoom V16 with PlanApo Z 1.5× lens equipped with Canon EOS r5 was used. Each stacked microphotography was created by stacking of 30-80 focal planes using the image stacking software ZereneStacker (https://zerenesystems.com/ $\mathrm{cms} /$ stacker). Each micrograph is equipped with the browsing software Krpano version 1.20 .8 (https://krpano.com/home/), which enables the observation of detailed structures of the object. Photos were finalized and edited in Zoner Photo Studio $\mathrm{X}$ (https://www.zoner.eu/) imaging software. The terminology used in this study is based on Kehlmaier (2005) and Skevington (2002) and follows recent taxonomic papers of the family (Kehlmaier et al. 2019, Motamedinia et al. 2020). 


\section{Results}

Pipunculus adami Kozánek, sp. nov.

Figs $1-18$

Type locality. Slovakia, Kamenica nad Hronom, 47 49'31.80"N, $18^{\circ} 44^{\prime} 55.02^{\prime \prime} \mathrm{E}$.

Material examined. Holotype. 1 male, SNM: Slovakia, Kamenica nad Hronom, 47 49 $31.80^{\prime \prime} \mathrm{N}, 18^{\circ} 44^{\prime} 55.02^{\prime \prime} \mathrm{E}$, 18.vii.2010, Malaise trap, leg. O. Majzlan and L. Vidlička. Paratypes. 9 males, 1 female, SNM: Slovakia, Kamenica nad Hronom, 47 49'31.80"N, 18 44'55.02"E, 11.vii.2010 (2 males, 1 female), 18.vii.2010 (3 males), 22.vii.2010 (2 males), 1.viii.2010 (2 males), Malaise trap, leg. O. Majzlan and L'. Vidlička.

Differential diagnosis. Pipunculus adami Kozánek, sp. nov. can be affiliated to the $P$. elegans species group by having the membranous area of syntergosternite 8 regularly ovate (not narrowing in the lower part), by its reduced pollinosity on the scutum and abdominal tergites, and by the phallus with its ejaculatory ducts being short and straight. Males of $P$. adami Kozánek, sp. nov. can be separated from the rest of European P. elegans species group by its large membranous area, being more roundish than ovate, the broad and blunt surstyli, the broad phallic guide, and by the hind tibiae with 4-5 long light setae anteromedially. Female can be distinguished by the following characters: pulvilli long, base of ovipositor rather robust, dorsally semiglobular, piercer straight, rather stout and short, about as long as $1.5 \times$ length of base.

Description of male. Head (Fig. 1, Fig. 2, Fig. 3). Frons densely silver-grey pollinose, medially with indistinct spot of reduced pollinosity. Face silver-grey pollinose. Ocellar triangle black, equilateral. Occiput greyish pollinose, in upper margin with reduced pollinosity, with narrow strip of brownish pollinosity behind ocellar triangle. Eyes meeting for distance more or less equal to the length of frons (17-18 facets). Frontal facets of compound eyes not enlarged. Scape and pedicel with brown pollinosity. Pedicel dorsally and ventrally with 4-5 light long bristles. Flagellum brown pollinose, blunt. Arista dark brown, posterior side of its broadened base brownish pollinose.

Thorax. Postpronotal lobe dark brown to black, greyish pollinose. Prescutum laterally greyish pollinose, medially matt black. Scutum in front one-third to one-half laterally greyish, medially brownish pollinose, posteriorly shining black. Scutellum shining black laterally with greyish pollinosity with row of rather dense and long whitish hairs. Subscutellum black with greyish pollinosity. Pleura dark with greyish pollinosity. Wing clear, transparent, covered with microtrichia, which can be reduced to various degree in medial cells (cup, bm, br). Pterostigma present. Crossvein $\mathrm{r}-\mathrm{m}$ reaches cell $\mathrm{dm}$ shortly behind one-quarter of cell length. Vein $\mathrm{M}_{1+2}$ undulating (Fig. 4). For wing measurements see Tab. 1. Halter light, basally dark brown. Coxa black, greyish pollinose. Trochanter dark brown. Femur black, yellowish in apical quarter. Tibia dark brown, yellowish in basal third, anteromedially with 4-5 long light setae. Tarsus dark. Pulvillus as long as or slightly shorter than distitarsus (Fig. 5, Fig. 6).

Abdomen. Tergite 1 entirely greyish pollinose, laterally with tuft of 10-12 long light bristles. Terga 2-5 shining, black with sparse longer light hairs, posterolaterally with greyish pollinosity. Terga $2-4$ equally long, tergum 5 about $1.5 \times$ longer than any previous tergite (Fig. 7, Fig. 8). Syntergosternite 8 subshining black, brownish pollinose. Membraneous area large, roundish
(Fig. 9). Surstyli broad, irregular, and strongly curved (Fig. 10). Right surstylus longer, left surstylus short, simple (Fig. 11, Fig. 12). Phallic guide rather broad (compared with other species of $P$. elegans group), sharply pointed, phallus trifid, ejaculatory ducts short (Fig. 13).

Description of female. Head (Fig. 14, Fig. 15, Fig. 16). Frons subparallel, distinctly narrowing in hind one-third. Frons greyish pollinose, in lower half with subshining short gap-shaped patch, in upper half with subshining long M-shaped pattern. Ocellar triangle equilateral. Occiput uniformly densely greyish pubescent. Compound eyes with front 10-11 rows of enlarged facets. Scape and pedicel with dark brown pollinosity, pedicel dorsally and ventrally with 4-5 long dark bristles. Flagellum brown pollinose shortly acute. Arista brown, posterior side of its broadened base brownish pollinated.

Thorax. Postpronotal lobe densely greyish pollinose. Prescutum greyish pollinose medially with reduced pollinosity. Scutum shining black, narrowly greyish pollinose in anterior margin. Scutum covered with sparse whitish hairs. Anterior margin narrowly greyish pollinose, otherwise shining, black, covered with sparse light hairs. Subscutellum black with grey pollinosity. Pleura dark greyish pollinose. Wing clear, transparent, covered with microtrichia. Pterostigma present. Crossvein $\mathrm{r}-\mathrm{m}$ reaches cell $\mathrm{dm}$ shortly behind one-quarter of cell length. Vein $\mathrm{M}_{1+2}$ undulating. For wing measurements see Tab. 1. Coxa black, greyish pollinose. Trochanter light brown. Femur black narrowly yellow at base and apex. Tibia dark, yellowish in basal quarter. Tarsus dark. Pulvillus longer than distitarsus.

Abdomen. Tergite 1 greyish pollinose laterally with fringe of long light bristles. Terga 2-6 shining, black, sparsely covered with light hairs. Terga $2-5$ posterolaterally and lateral margin of tergum 6 greyish pollinose. Base of ovipositor semi-ovoid, shining, dark brown to black, sparsely pollinose in anterior margin. Piercer dark, straight, continually is tapering to the apex (Fig. 17, Fig. 18).

Etymology. The species name is dedicated to the grandson of the first author - Adam Strečko.

Pipunculus anatolicus Kozánek, sp. nov.

Figs 19-27

Type locality. Turkey, Mugla, university campus, $37^{\circ} 09^{\prime} 38^{\prime \prime} \mathrm{N}$, $28^{\circ} 22^{\prime} 11^{\prime \prime} \mathrm{E}$.

Material examined. Holotype. 1 female, SNM: Turkey, Mugla, Univ. campus, $37^{\circ} 09^{\prime} 38^{\prime \prime} \mathrm{N}, 28^{\circ} 22^{\prime} 11^{\prime \prime} \mathrm{E}, 730 \mathrm{~m}$ a.s.l., xi.2015-iv.2016, Malaise trap, leg. M. Barták and Š. Kubík. Paratypes. 1 female, SNM: Turkey, Mugla, Univ. campus, $37^{\circ} 09^{\prime} 38^{\prime \prime} \mathrm{N}, 28^{\circ} 22^{\prime} 11^{\prime \prime} \mathrm{E}, 730 \mathrm{~m}$ a.s.1., xi.2015-iv.2016, Malaise trap, leg. M. Barták and Š. Kubík. 2 males, SNM: Turkey, Mugla, Univ. campus, $37^{\circ} 09^{\prime} 39^{\prime \prime} \mathrm{N}, 28^{\circ} 22^{\prime} 20^{\prime \prime} \mathrm{E}, 710 \mathrm{~m}$ a.s.1., xi.2012-iii.2013, Malaise trap, leg. M. Barták and Š. Kubík.

Differential diagnosis. Pipunculus anatolicus Kozánek, sp. nov. is described on the female specimens only. It can be affiliated to the $P$. elegans species group having scutum with reduced pollinosity. From females of the remaining representatives of this species group, it can be distinguished by a very short, straight piercer, which is about as long as the base of ovipositor, and by vein $\mathrm{M}_{1+2}$ strongly undulating.

Desription of female. Head (Fig. 19, Fig. 20, Fig. 21). Frons sub-parallel, slightly converging in upper half. Frons greyish pollinose. Anterior third of frons densely pollinose, pollinosity is continually reduced, with shining narrow medial line 

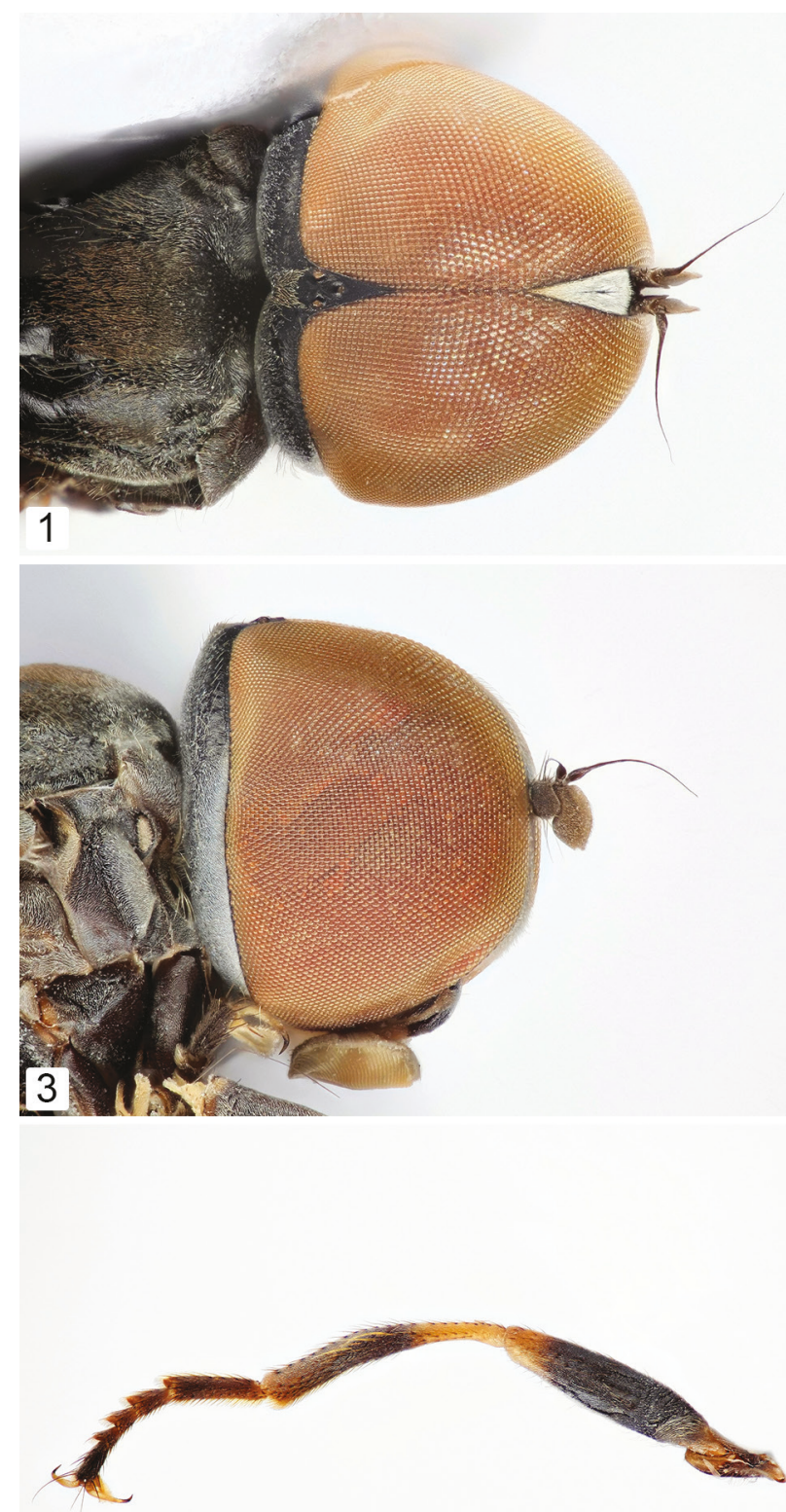

5

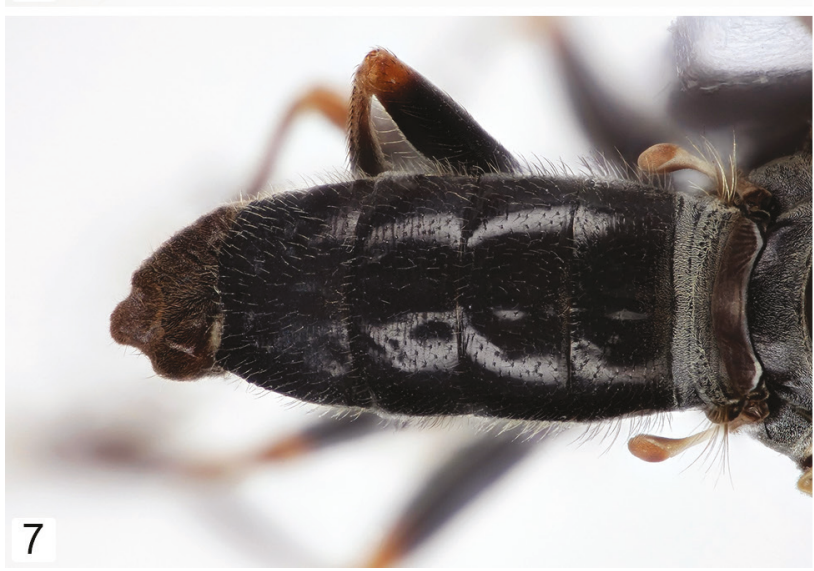

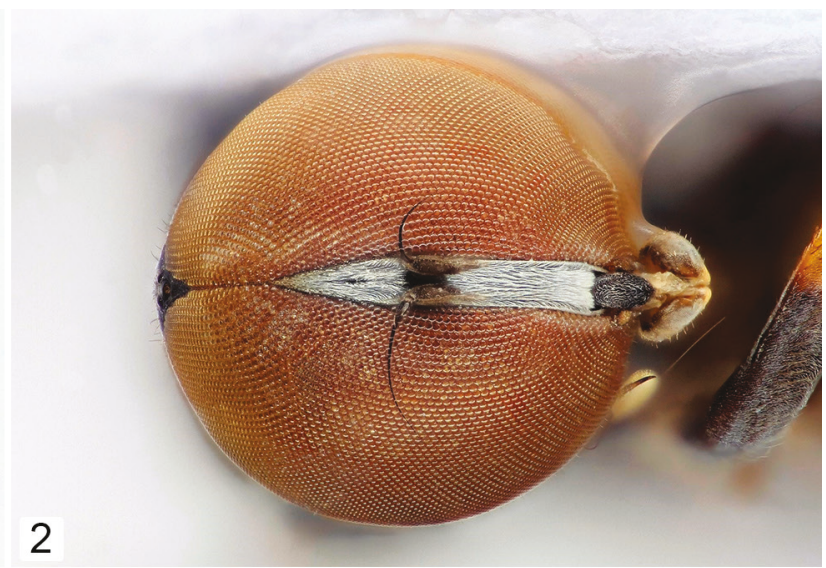
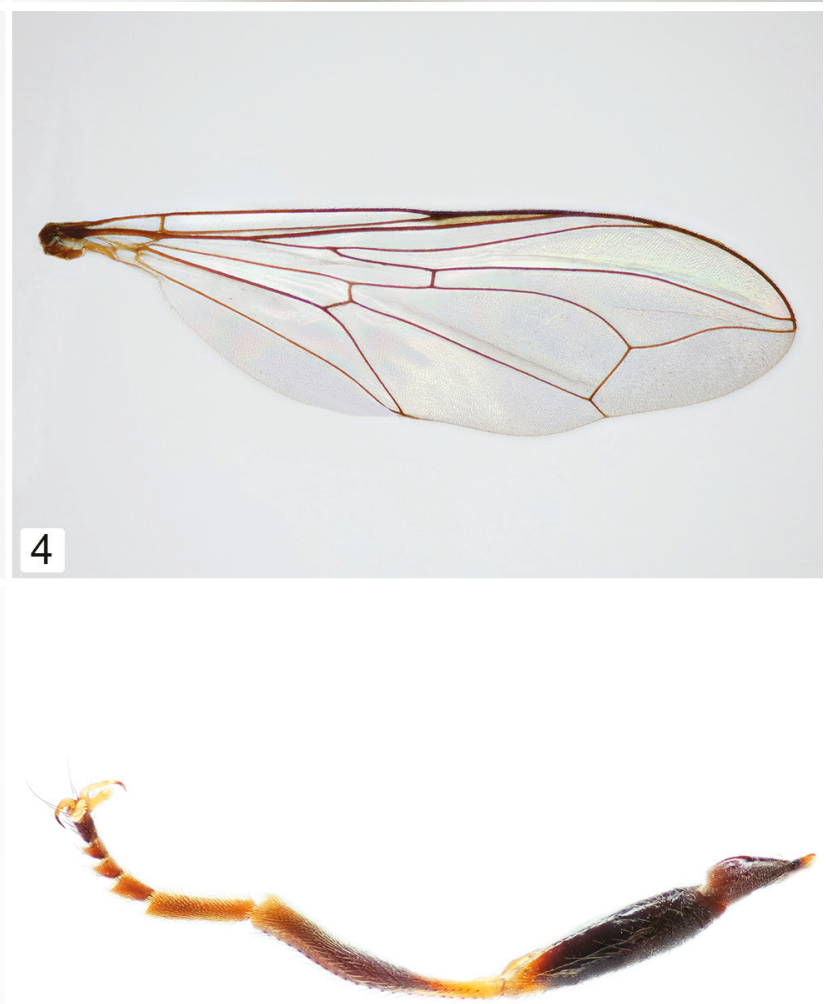

6

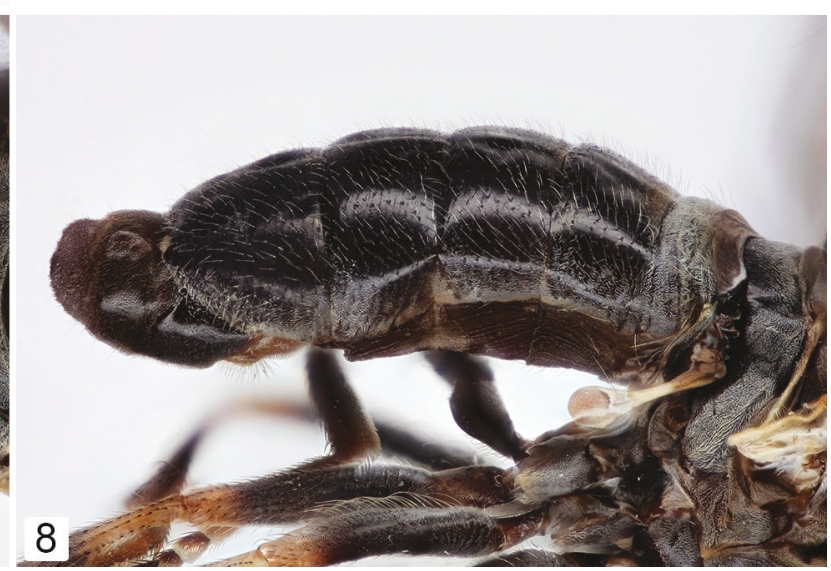

Figures 1-8. Details on diagnostic characters. Fig. 1. Pipunculus adami Kozánek, sp. nov., male head, dorsal view. Fig. 2. P. adami Kozánek, sp. nov., male head, frontal view. Fig. 3. P. adami Kozánek, sp. nov, male head, lateral view. Fig. 4. P. adami Kozánek, sp. nov., male right wing. Fig. 5. P. adami Kozánek, sp. nov., male right hind leg, anterior view. Fig. 6. P. adami Kozánek, sp. nov., male right hind leg, posterior view. Fig. 7. P. adami Kozánek, sp. nov., male abdomen, dorsal view. Fig. 8. P. adami Kozánek, sp. nov., male abdomen, lateral view. 

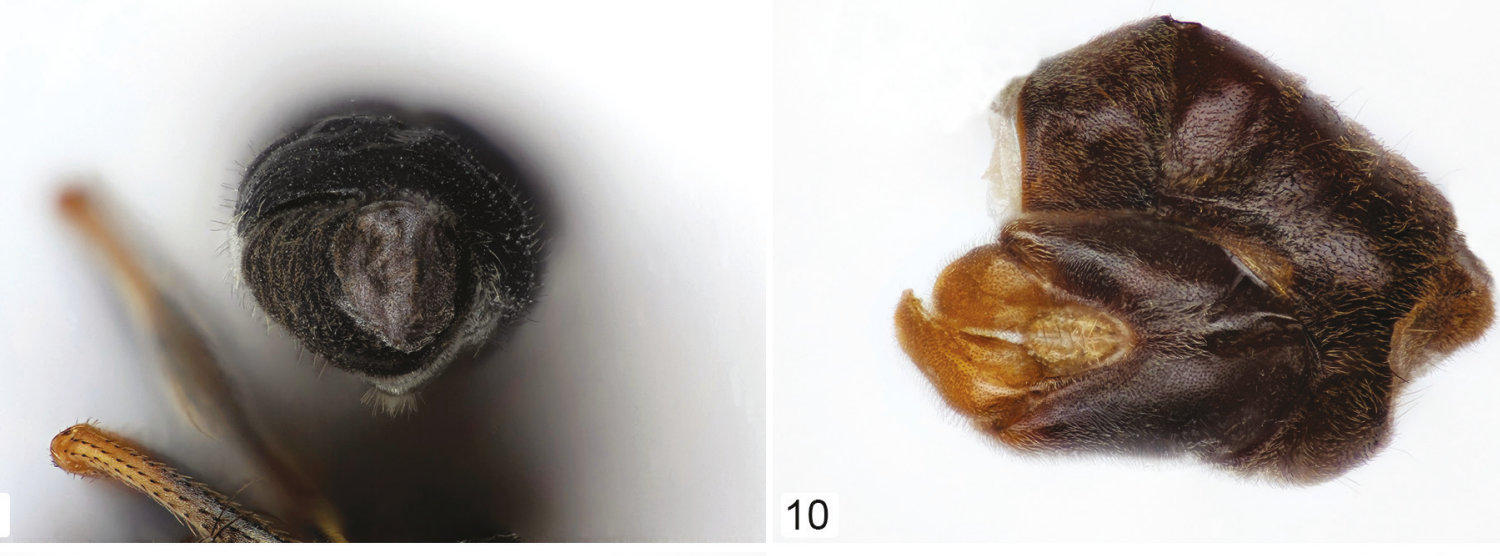

10
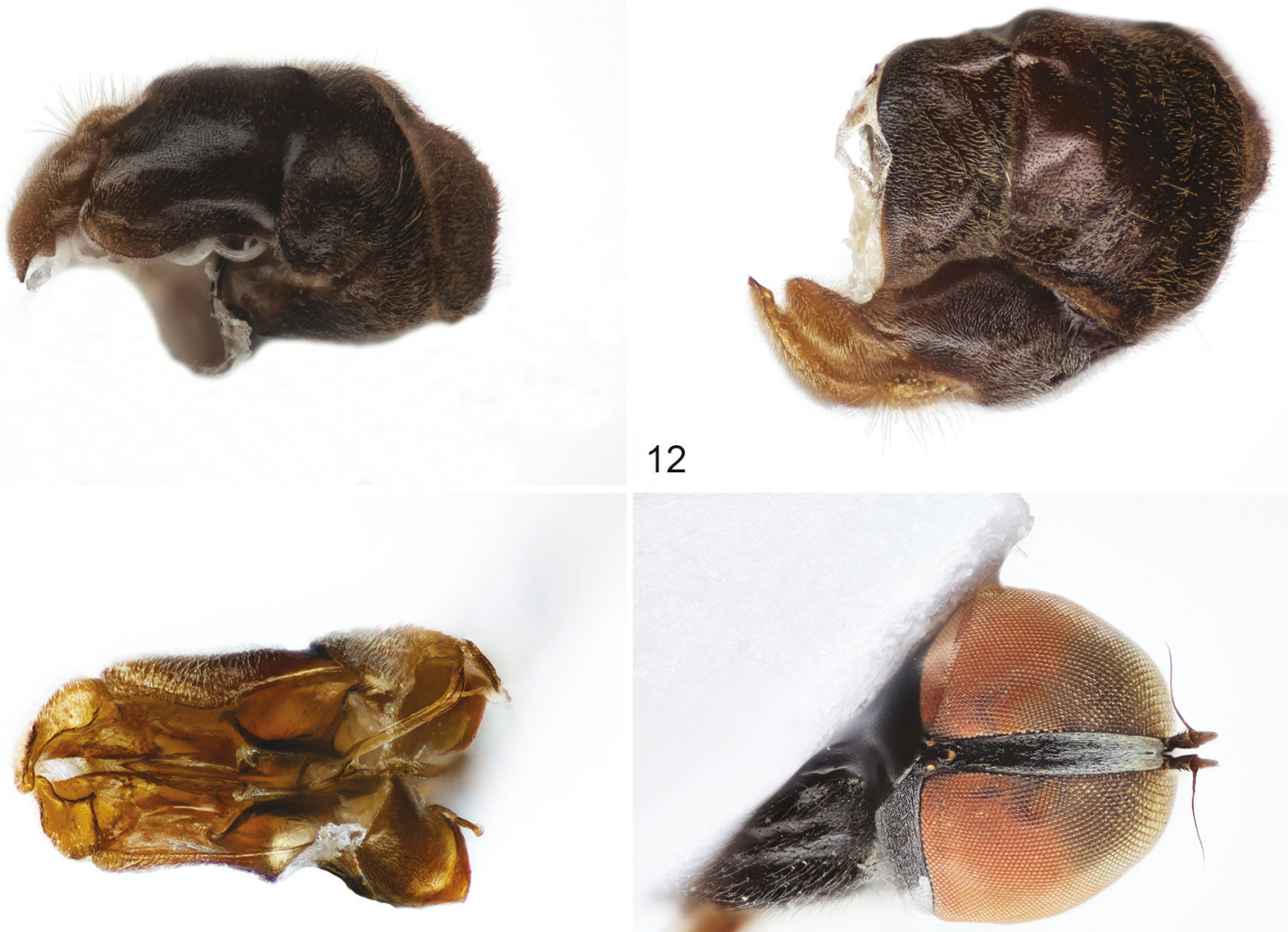

13
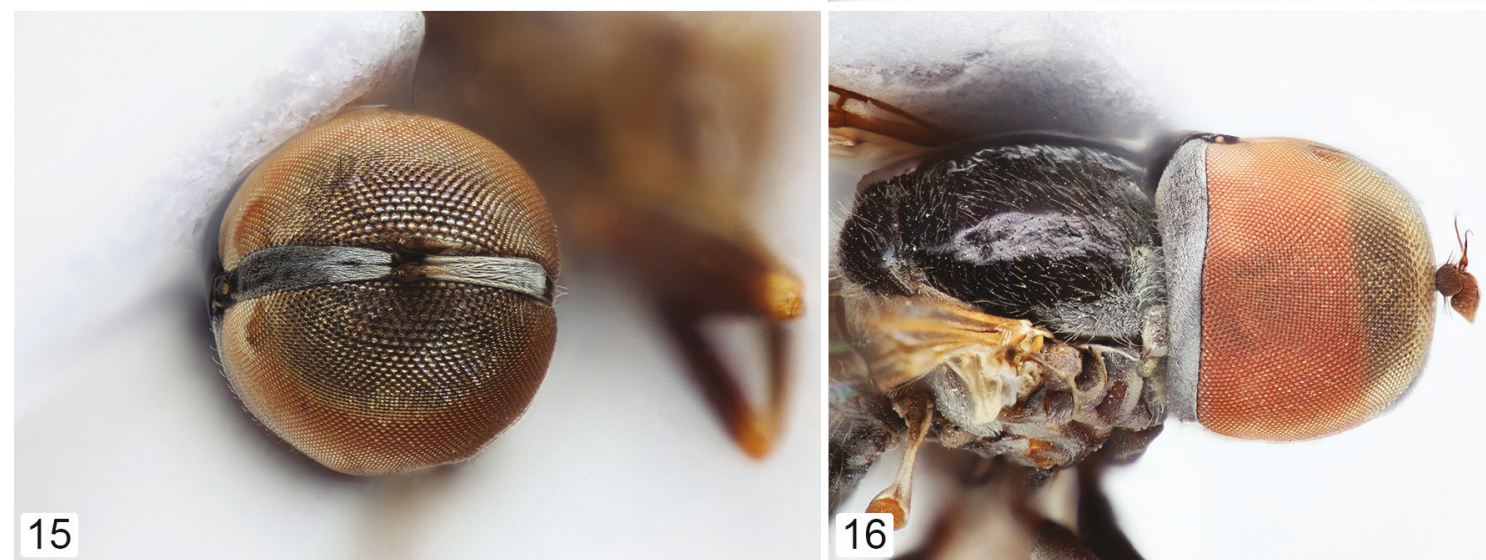

Figures 9-16. Details on diagnostic characters. Fig. 9. Pipunculus adami Kozánek, sp. nov., male syntergosternite 8, caudal view. Fig. 10. P. adami Kozánek, sp. nov., male epandrium, dorsal view. Fig. 11. P. adami Kozánek, sp. nov, male epandrium, left lateral view. Fig. 12. P. adami Kozánek, sp. nov., male epandrium, right lateral view. Fig. 13. P. adami Kozánek, sp. nov., male hypandrium, ventral view. Fig. 14. P. adami Kozánek, sp. nov., female head, dorsal view. Fig. 15. P. adami Kozánek, sp. nov., female head, frontal view. Fig. 16. P. adami Kozánek, sp. nov., female head, lateral view, lateral view. 

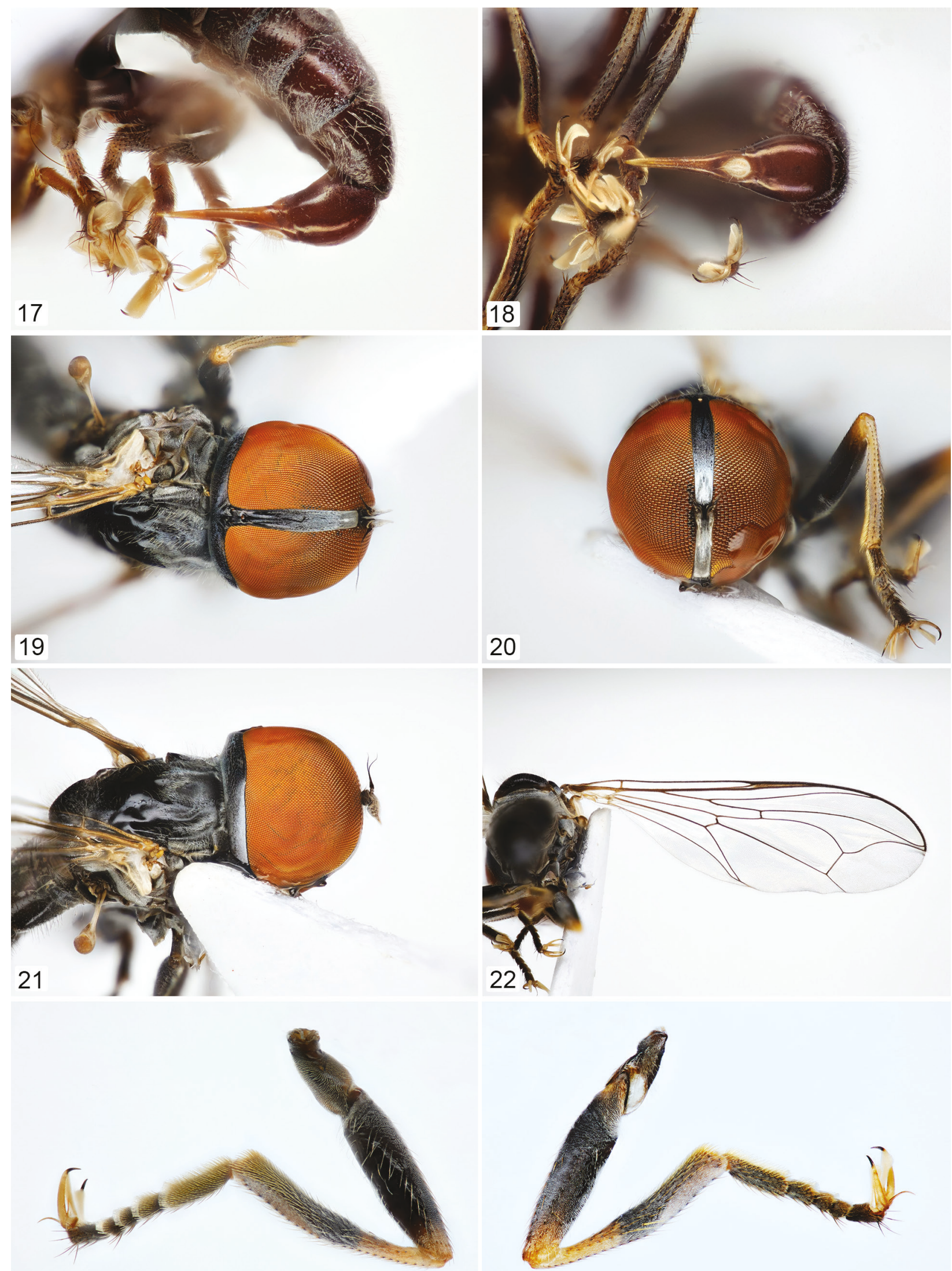

Figures 17-24. Details on diagnostic characters. Fig. 17. Pipunculus adami Kozánek, sp. nov., ovipositor, lateral view. Fig. 18. P. adami Kozánek, sp. nov., ovipositor, dorsal view. Fig. 19. P. anatolicus Kozánek, sp. nov, female head, dorsal view. Fig. 20. P. anatolicus Kozánek, sp. nov., female head, frontal view. Fig. 21. P. anatolicus Kozánek, sp. nov., female head, lateral view. Fig. 22. P. anatolicus Kozánek, sp. nov., female right wing. Fig. 23. P. anatolicus Kozánek, sp. nov., female right hind leg, posterior view. Fig. 24. P. anatolicus Kozánek, sp. nov., female right hind leg, anterior view. 
Table 1. Morphometric data for key characters measured on four Pipunculus Latreille, 1802 species. Measured values are given in millimeters.

\begin{tabular}{|c|c|c|c|c|c|}
\hline & \multicolumn{2}{|c|}{ Pipunculus adami sp. nov. } & \multirow{2}{*}{$\begin{array}{c}\text { P. anatolicus sp. nov. } \\
\text { Females }\end{array}$} & \multirow{2}{*}{$\begin{array}{l}\text { P. lindae sp. nov. } \\
\text { Females }\end{array}$} & \multirow{2}{*}{$\begin{array}{c}\text { P. wolfi Kovarz, } 1887 \\
\text { Males }\end{array}$} \\
\hline & Males & Females & & & \\
\hline$N$ & 8 & 1 & 3 & 2 & 4 \\
\hline \multicolumn{6}{|l|}{ Head } \\
\hline Length of frons & $0.43-0.47$ & 0.45 & $1.08-1.14$ & $1.01-1.06$ & $0.48-0.52$ \\
\hline Eyes touching distance & $0.47-0.54$ & & & & $0.33-0.38$ \\
\hline Length of ocellar triangle & $0.09-0.11$ & & & & $0.12-0.14$ \\
\hline Width of frons above antennae & 0.05 & & $0.13-0.15$ & 0.11 & \\
\hline Maximal width of frons & 0.08 & & $0.18-0.19$ & $0.15-0.17$ & \\
\hline Length of flagellum & $0.11-0.13$ & & $0.27-0.3$ & $0.25-0.26$ & \\
\hline Width of flagellum & $0.06-0.07$ & & $0.1-0.11$ & $0.09-0.1$ & \\
\hline \multicolumn{6}{|l|}{ Right Wing } \\
\hline Length of wing & $4.84-5.42$ & 4.1 & $4.8-5.09$ & $4.29-4.41$ & $5.34-5.61$ \\
\hline Width of wing & 1.26 & $1.42-1.45$ & 1.42 & $1.52-1.73$ & $1.48-1.68$ \\
\hline Length of 3 rd costal section & $0.98-1.04$ & 0.66 & $1.02-1.08$ & $0.44-0.45$ & $0.99-1.17$ \\
\hline Length of 4 th costal section & $0.63-0.74$ & 0.39 & $0.56-0.65$ & $0.52-0.56$ & $0.65-0.8$ \\
\hline \multicolumn{6}{|l|}{ Abdomen } \\
\hline Length of 5th tergite & $0.59-0.68$ & & & & $0.66-0.71$ \\
\hline Width of 5th tergite & $0.97-1.06$ & & & & $0.89-1.03$ \\
\hline Length of syntergosternite 8 & $0.34-0.43$ & & & & $0.59-0.63$ \\
\hline Width of syntergosternite 8 & $0.69-0.79$ & & & & $0.73-0.78$ \\
\hline Length of epandrium & $0.5-0.63$ & & & & $0.48-0.62$ \\
\hline Width of epandrium & $0.42-0.54$ & & & & $0.35-0.39$ \\
\hline Total length of ovipositor & 1.54 & & $0.78-0.81$ & $1.17-1.22$ & \\
\hline Length of ovipositor base & 0.86 & & $0.41-0.44$ & $0.5-0.52$ & \\
\hline Length of piercer & 0.68 & & $0.36-0.4$ & $0.65-0.68$ & \\
\hline
\end{tabular}

bellow frontal triangle and laterally with arrow shaped pattern of reduced pollinosity. Ocellar triangle subshining black, equilateral. Occiput in upper one-third subshining black with sparse greyish pollinosity, its lower half densely grey pollinose. Compound eyes with $15-17$ front rows of moderately enlarged facets. Scape and pedicel dark, brown pollinose, pedicel dorsally with 3-4 shorter ventrally with 5-6 longer light bristles. Flagellum brownish, marginally greyish pollinose, acuminate. Arista black.

Thorax. Postpronotal lobe dark, greyish pollinose. Prescutum laterally dense, medially more sparsely greyish pollinose. Scutum shining black with greyish pollinosity in anterior onethird and along posterior margin. Postpronotal lobes, prescutum, and anterior one-third of scutum with longer light hairs. Subscutellum black, greyish pollinose. Scutellum shining black with sparse long light hairs. Pleura with dense greyish pollinosity. Wing clear, transparent, covered with microtrichia which are reduced in medial cells (cup, bm, br). Pterostigma present. Crossvein $\mathrm{r}-\mathrm{m}$ reaches cell $\mathrm{dm}$ shortly behind one-quarter of cell length. Vein $\mathrm{M}_{1+2}$ strongly undulated (Fig. 22). For wing measurements see Tab. 1. Halter light, basally darkened, halter knob light brown. Coxa black, sparsely greyish pollinose. Trochanter dark brown. Femur black, narrowly yellowish at base and apex. Tibia dark brown, yellowish in basal one-third to onehalf. Hind tibiae mediolaterally with with 3-4 stronger light setae. Tarsus black. Pulvillus is slightly longer than distitarsus (Fig. 23, Fig. 24).

Abdomen. Tergite 1 greyish pollinose, laterally with fringe of long white bristles. Tergite 2 in anterodorsal one-third with sparse brownish pollinosity. Terga 2-6 shining, black, narrowly greyish pollinose in lateral margins (Fig. 25, Fig. 26). Base of ovipositor cylindrical, black, with sparse light pollinosity. Pos- terior margin of base shining dark brown. Piercer short, slim, stiletto shaped, brownish (Fig. 27).

Etymology. The species name is derived from the historical name of the country of species origin (Anatolia).

\section{Pipunculus lindae Kozánek, sp. nov.}

Figs 28-36

Type locality. Slovakia, Krížna dolina, Vysoké Tatry Mts, $49^{\circ} 09^{\prime} 28.44^{\prime \prime} \mathrm{N}, 19^{\circ} 56^{\prime} 41.58^{\prime \prime} \mathrm{E}, 1196 \mathrm{~m}$ a.s.1.

Material examined. Holotype. 1 female, SNM: Slovakia, Vysoké Tatry Mts, Krížna dolina, 49 $09^{\prime} 28.44^{\prime \prime} \mathrm{N}, 19^{\circ} 56^{\prime} 41.58^{\prime \prime} \mathrm{E}$, 1196 m a.s.1., 29.vi.2009, MT, leg. O. Majzán and L. Vidlička. Paratype. 1 female, SNM: Czech Republic, Šumava Mts., Rokytecká slat', forest, $49^{\circ} 00^{\prime} 59^{\prime \prime} \mathrm{N}, 13^{\circ} 25^{\prime} 05^{\prime \prime} \mathrm{E}, 1100 \mathrm{~m}$ a.s.l., 16.vi.-21.vii.1999, Malaise trap, leg. M. Barták.

Differential diagnosis. Pipunculus lindae Kozánek, sp. nov. belongs to the $P$. campestris species group. The species resembles P. lichtwardti Kozánek, 1981 (Fig. 37, Fig. 38), from which it differs in mesonotum being anterolaterally greyish pollinose with no admixture of brown pollinosity, terga 4-6 dorsally shinning black at most with very narrow strip of pollinosity on anterior margin, shape of ovipositor with base cylindrical and piercer sharply separated from base.

Description of female. Head (Fig. 28, Fig. 29, Fig. 30). Frons sub-parallel, slightly converging in upper half, greyish pollinose. Posterior one-third with pattern consisting of narrow medial line and broader lateral stripes of reduced pollinosity. Ocellar triangle subshining black, equilateral. Occiput uniformly greyish pubescent with narrow strip of brownish pubescence behind ocellar triangle. Compound eyes with 10-12 front rows of moderately enlarged facets. Scape light brown, in upper mar- 

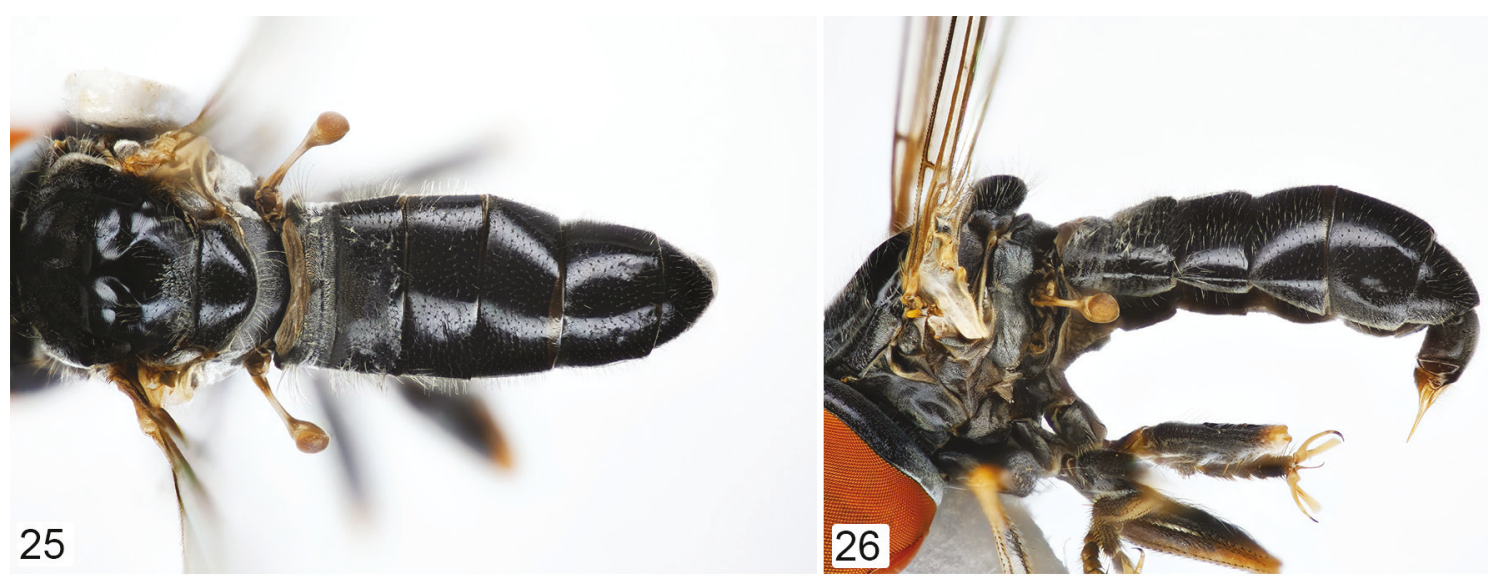

27
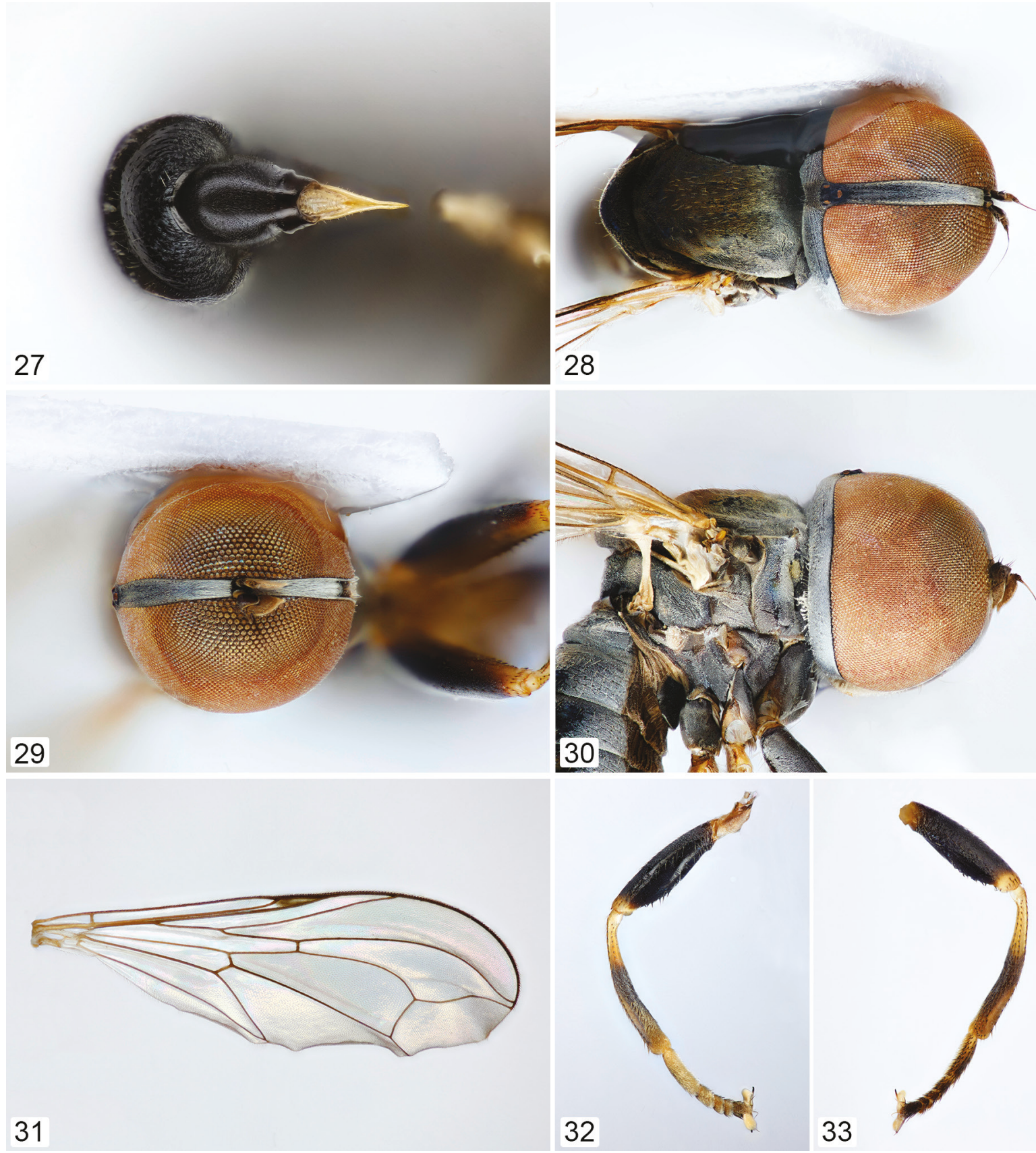

Figures 25-33. Details on diagnostic characters. Fig. 25. Pipunculus anatolicus Kozánek, sp. nov., female abdomen, dorsal view. Fig. 26. P. anatolicus Kozánek, sp. nov., female abdomen, lateral view. Fig. 27. P. anatolicus Kozánek, sp. nov, ovipositor, dorsal view. Fig. 28. P. lindae Kozánek, sp. nov., female head, dorsal view. Fig. 29. P. lindae Kozánek, sp. nov., female head, frontal view. Fig. 30. P. lindae Kozánek, sp. nov., female head, lateral view. Fig. 31. P. lindae Kozánek, sp. nov., female right wing. Fig. 32. P. lindae Kozánek, sp. nov., female right hind leg, posterior view. Fig. 33. P. lindae Kozánek, sp. nov., female right hind leg, anterior view. 

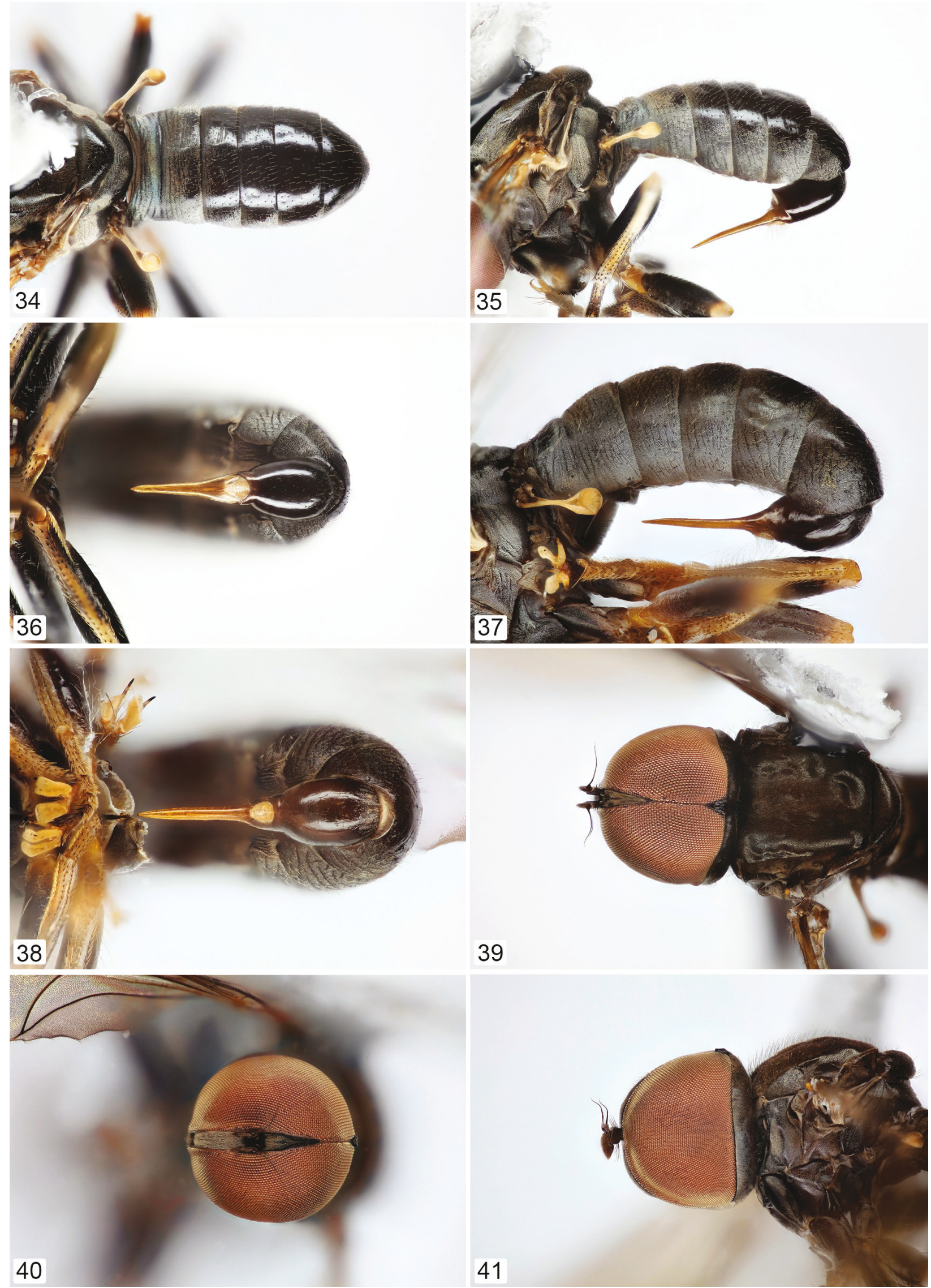

Figures 34-41. Details on diagnostic characters. Fig. 34. Pipunculus lindae Kozánek, sp. nov., female abdomen, dorsal view. Fig. 35. P. lindae Kozánek, sp. nov., female abdomen, lateral view. Fig. 36. P. lindae Kozánek, sp. nov., female ovipositor, dorsal view. Fig. 37. P. lichtwardti Kozánek, 1981, female abdomen, lateral view. Fig. 38. P. lichtwardti Kozánek, 1981, female ovipositor, dorsal view. Fig. 39. P. wolfi Kowarz, 1887, male head, dorsal view. Fig. 40. P. wolfi Kowarz, 1887, male head, frontal view. Fig. 41. P. wolfi Kowarz, 1887, male head, lateral view. 


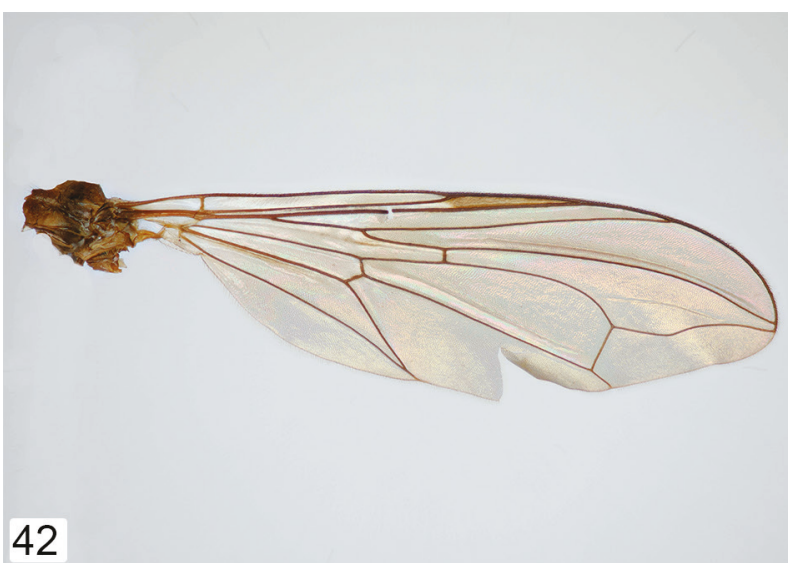

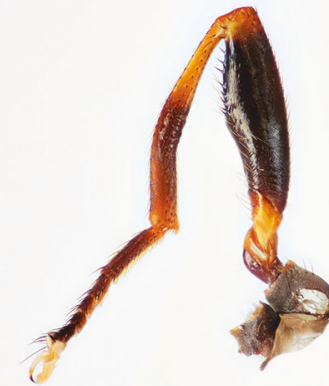

43

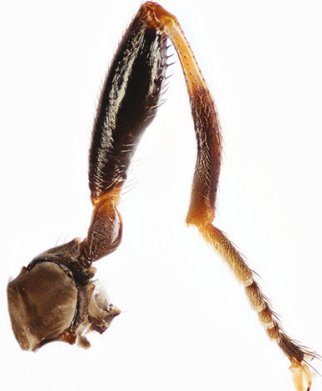

44

45
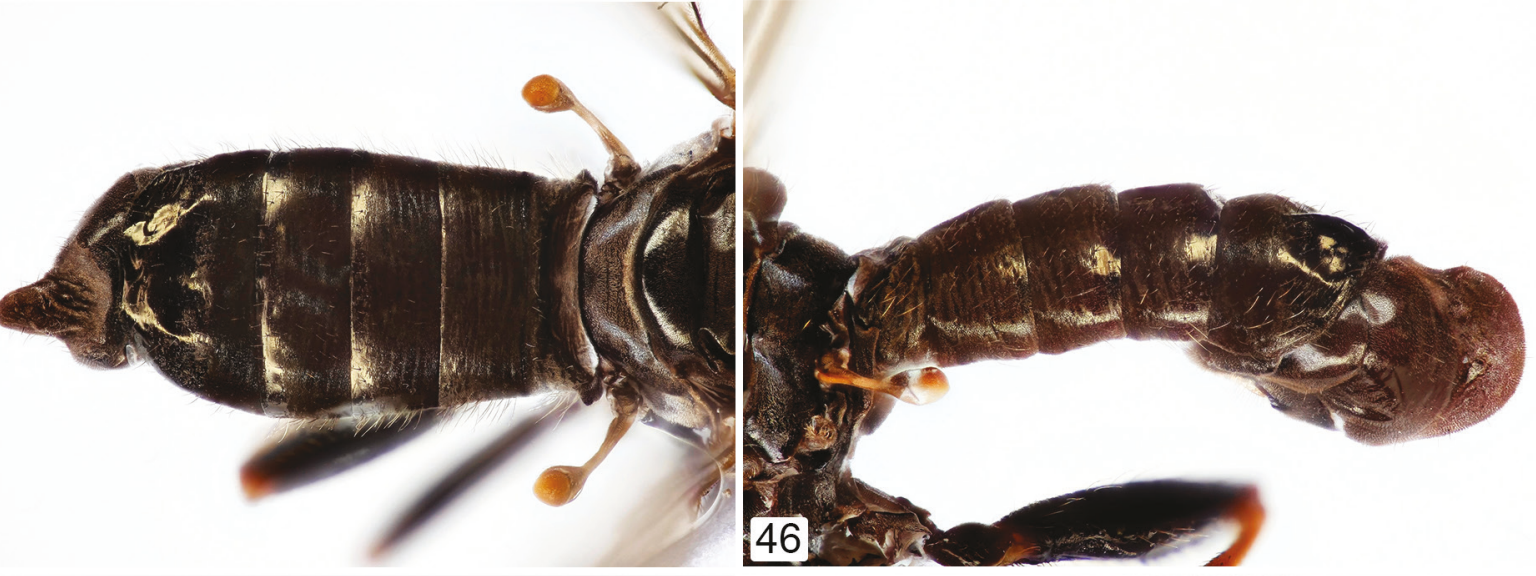

47
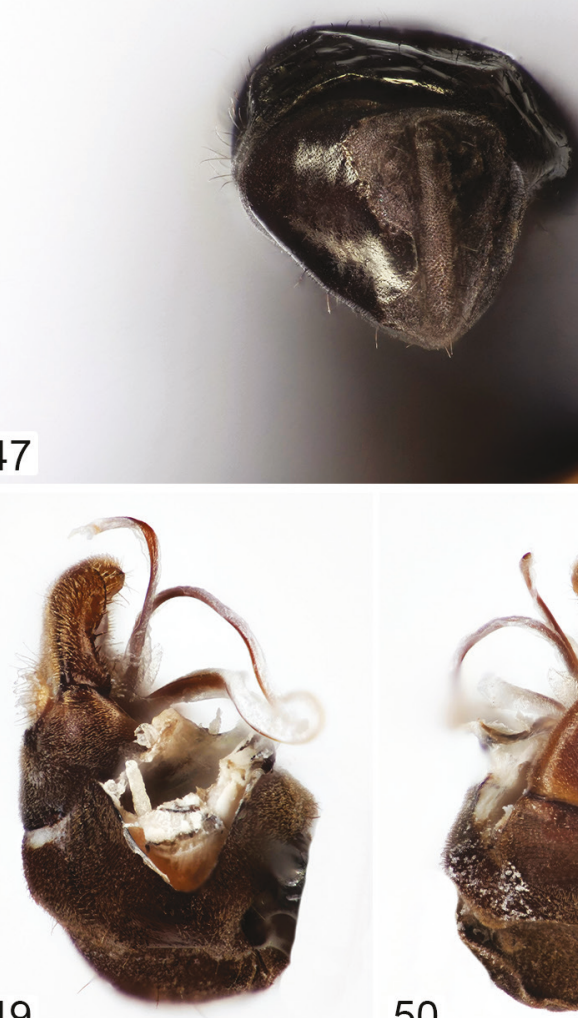

49
50
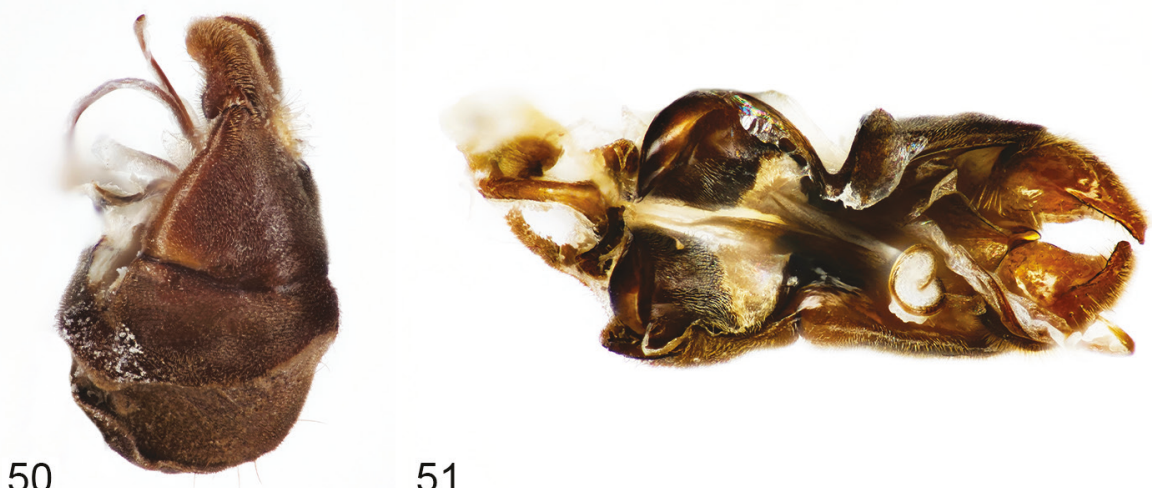

51

Figures 42-51. Details on diagnostic characters. Fig. 42. Pipunculus wolfi Kowarz, 1887, male right wing. Fig. 43. P. wolfi Kowarz, 1887, male right hind leg, anterior view. Fig. 44. P. wolf Kowarz, 1887, male right hind leg, posterior view. Fig. 45. P. wolfi Kowarz, 1887, male abdomen, dorsal view. Fig. 46. P. wolfi Kowarz, 1887, male abdomen, lateral view. Fig. 47. P. wolfi Kowarz, 1887, male syntergosternite 8, caudal view. Fig. 48. P. wolfi Kowarz, 1887, male epandrium, dorsal view. Fig. 49. P. wolf Kowarz, 1887, male epandrium, left view. Fig. 50. P. wolfi Kowarz, 1887, male epandrium, right view. Fig. 51. P. wolfi Kowarz, 1887, male hypandrium, ventral view. 
gin with dark seta. Pedicel dark brown to black, in upper half with four dark brown setae, in lower half brown. Inner side of pedicel shining brown. Flagellum light brown, shortly acuminate. Arista shining brown, base dorsally brownish pollinose.

Thorax. Postpronotal lobe dark, densely greyish pubescent. Mesonotum anteriorly and laterally greyish pubescent with sparse longer light hairs, brownish pubescent on disc and hind margin. All pleural sclerites with greyish pubescence. Scutellum subshining black with sparse brownish pollinosity in anterior margin. Hind margin of scutellum with few shorter light hairs. Subscutellum densely greyish pubescent. Wing clear, transparent, covered with microtrichia which are reduced along cells $\mathrm{bm} / \mathrm{br}$ common section of vein $\mathrm{M}$. Pterostigma present. Crossvein $\mathrm{r}-\mathrm{m}$ reaches cell $\mathrm{dm}$ at basal one-third of cell length. Vein $\mathrm{M}_{1+2}$ distinctly undulating (Fig. 31). For wing measurements see Tab. 1. Trochanter yellowish. Femur black, narrowly yellow at base and apex. Tibia dark, yellowish in basal one-quarter and along anterior margin. Tarsus dark. Pulvilli slightly longer than distitarsus (Fig. 32, Fig. 33).

Abdomen. Tergite 1 greyish pollinose medially with admixture of brownish pollinosity. Tergite 2 subshining black, in anterior half with silver, medially brownish, pollinosity. Tergite 3 narrowly greyish-brown pollinose along anterior margin. Terga 2-6 broadly greyish pubescent laterally (Fig. 34, Fig. 35). Base of ovipositor shining black, sharply separated from long straight light brown piercer (Fig. 36).

Etymology. The species name is dedicated to the granddaughter of the first author - Linda Strečková.

\section{Pipunculus wolfi Kowarz, 1887}

Figs 39-51

\section{Pipunculus wolfi Kowarz, 1887: 152}

Material examined. 1 male, 1 female, SNM: Czech Republic, Šumava Mts, Nová Hůrka, spruce forest, $49^{\circ} 09^{\prime} 21^{\prime \prime} \mathrm{N}$, $13^{\circ} 20^{\prime} 00^{\prime \prime}$ E, $880 \mathrm{~m}$ a.s.1., 24.vi.-28.vii.2000, Malaise trap, leg. M. Barták. 1 male, SNM: Czech Republic, Krkonoše Mts, Zrcadlový potok, nr. brook, $50^{\circ} 38^{\prime} 1.9^{\prime \prime} \mathrm{N}, 14^{\circ} 43^{\prime} 54.6^{\prime \prime} \mathrm{E}, 670 \mathrm{~m}$ a.s.1., 1.ix.-13.x.2009, Malaise trap, leg. J. Vaněk. 1 female, SNM: Czech Republic, Krkonoše Mts, Upská jáma (Obří důl), $50^{\circ} 44^{\prime} 0.8^{\prime \prime} \mathrm{N}, 15^{\circ} 43^{\prime} 32^{\prime \prime}, 1100$ m a.s.1., Malaise trap, leg. J. Vaněk. 1 male, SNM: Czech Republic, Vráž u Písku, damp meadow, $400 \mathrm{~m}$ a.s.1., $49^{\circ} 24^{\prime} 13^{\prime \prime} \mathrm{N}, 14^{\circ} 07^{\prime} 15^{\prime \prime} \mathrm{E}, 400 \mathrm{~m}$ a.s.1., 31.v.-4. vi.2004, Malaise trap, leg. M. Barták. 1 female, SNM: Czech Republic, Vráž u Písku, damp meadow, $400 \mathrm{~m}$ a.s.1., $49.23^{\circ} \mathrm{N}$, 14.08ㅌ, 27.v.-2.vi.1996, Malaise trap, leg. M. Barták.

Differential diagnosis. This large species is related to Pipunculus lenis Kuznetzov, 1991, from which it differs in the following characters: abdominal terga laterally with no trace of grey pollinosity, brownish pollinose, surstyli broad, shortly pointed, base of phallic guide very broad.

Redescription of male. Head (Fig. 39, Fig. 40, Fig. 41). Frons densely light brown pollinose, in upper part with minute frontolateral hairs. Frontal patch posteriorly long acuminate, in lower half brownish in upper brownish-grey pollinose, medially with indistinct gap of reduced pollinosity. Face light brown pollinose. Ocellar triangle shining black, equilateral. Occiput greyish pollinose, in upper one-third and narrowly in hind margin with brown pollinosity. Eyes meeting for distance more or less equal to the length of frons (17-18 facets). Frontal facets of compound eyes not enlarged. Scape and pedicel dark brown.
Flagellum brown, acute. Pedicel dorsally and ventrally with 4-5 long bristles. Arista black, posterior side of broadened base pollinose.

Thorax. Postpronotal lobe dark with lighter upper margin. Prescutum, scutum, scutellum and subscutellum black. Prescutum and scutum with two mediolateral rows of long dark bristles, brown pollinose, laterally with grey pollinosity. Scutellum brown pollinose. Subscutellum brown pollinose, laterally with admixture of grey pollinosity. Pleura dark, grey pollinose with admixture of brown pollinosity. Wing clear, transparent, very slightly brownish infuscated, uniformly covered with microtrichia. Pterostigma present. Crossvein $\mathrm{r}-\mathrm{m}$ reaches cell $\mathrm{dm}$ shortly behind quarter of its length. Vein $\mathrm{M}_{1+2}$ undulating (Fig. 42). For wing measurements see Tab. 1. Halter with light brown knob. Coxae dark, grey pollinose. Trochanter brown. Femur black, narrowly yellowish at base and apex. Hind femur in apical onethird with double row of $8-9$ spines. Tibia brown, yellowish in basal third, light brown at apex. Tarsus dark brown. Pulvilli as long as distitarsus (Fig. 43, Fig. 44).

Abdomen. Tergite 1 dark, brownish pollinose, laterally with tuft of 10-12 long light bristles. Terga 2-4 brownish pollinose, narrowly shining black at hind margin. Tergum 5 brownish pollinose anterior half, otherwise shining black. All terga with sparse, long, light hairs (Fig. 45, Fig. 46). Syntergosternite 8 subshining black, sparsely brown pollinose. Membranous area large, key-hole shaped with distinct keel (Fig. 47). Surstyli broad, almost symmetrical, shortly tipped at the apex (Fig. 48, Fig. 49, Fig. 50). Aedeagal guide broad, shortly pointed. Aedeagus trifid, ductuli long (Fig. 51).

\section{Discussion}

The European species of the genus Pipunculus, with the emphasis on Central European fauna, were first summarized by Kozánek (1981) who also provided the key for species known at that time. Kuznetzov $(1991,1993)$ further described several new Pipunculus species mainly from the European part of Russia. In a study of Finnish Pipunculidae, Kehlmaier (2008) advanced our knowledge of European species of the genus $\mathrm{Pi}$ punculus to a qualitatively new level. He solved a number of systematic uncertainties, provided detailed descriptions of all European species, and developed a modern key widely using stable morphological characters mainly on external terminalia. Despite the progress achieved in the last decades, our knowledge on European Pipunculus taxonomy, distribution, and biology is far from complete. Deficiencies in knowledge of the local Pipunculidae fauna still persist and extensive faunistic research in Southern and Eastern European countries is still needed. Continuing faunistic research focused on the biodiversity mainly in conserved areas can reveal the existence of new rare species. Pipunculus adami Kozánek, sp. nov. and P. lindae Kozánek, sp. nov. described in this work were also collected as a part of a research program focused on the entomofauna of conserved areas in Slovakia (Kováčovské kopce NR and Vysoké Tatry NP) and the Czech Republic (Šumava NP).

A detailed visualization of morphological characters is an essential requirement for exact description of species as well as the development of determination keys. The effort for continuous improvement of visualization techniques is obvious also in Pipunculidae research. Schematic drawings were used as a gold standard technique for visualization of key pipunculid morpho- 
logical characters until Jervis (1992) in his revision of European Chalarus used scanning electron microscopy photographs. SEM photographs provide detailed information about the studied morphological characters but are difficult to use in routine taxonomic work. Stacked microphotography (stacking a series of focal planes taken in different depth of focus) provide visualization of the entire body or morphological characters and is increasingly used in pipunculid research (Kehlmaier et al. 2019, Motamedinia et al. 2020). Interactive photography, presented in this paper, is a fusion of high-resolution stacked microphotography and viewing software. It provides image quality comparable to SEM photographs. The interactive photography allows the observation of visualized morphological characters in detail. This novel method substantially increases the informative level of description and can also be effectively used in the creation of identification keys.

\section{Acknowledgements}

Authors would like to thank Dr. Christian Kehlmaier (Dresden, Germany) for his valuable comments on the manuscript, and Michal Kozánek, MD., PhD. (Fayetville, USA) for the English language reviewing.

\section{References}

Albrecht, A. 1990. Revision, phylogeny and classification of the genus Dorylomorpha (Diptera, Pipunculidae). Acta Zoologica Fennica, 188: 1-240.

De Meyer, M. 1989. The West-Palaearctic species of the pipunculid genera Cephalops and Beckerias (Diptera): classification, phylogeny and geographical distribution. Journal of Natural History, 23: 725-765.

Földvári, M. \& De Meyer, M. 1999. Revision of Central and West European Tomosvaryella Aczél species (Diptera, Pipunculidae). Acta Zoologica Academiae Scientiarum Hungaricae, 45: 299-334.

Jervis, M.A. 1992. A taxonomic revision of the pipunculid fly genus Chalarus Walker, with particular reference to the European fauna. Zoological Journal of the Linnaean Society, 105: 243-352.

Kehlmaier, C. 2005. Taxonomic revision of European Eudorylini (Insecta, Diptera, Pipunculidae). Verhandlungen des naturwissenschaftlichen Vereins in Hamburg (NF), 41: 45353.

Kehlmaier, C. 2006. The West-Palaearctic species of Jassidophaga Aczél and Verrallia Mik described up to 1966 (Diptera: Pipunculidae). Stuttgarter Beiträge zur Naturkunde, Serie A (Biologie), 697: 1-34.

Kehlmaier, C. 2008. Finnish Pipunculidae (Diptera) studies. Part 1: Taxonomic notes on Cephalops Fallén, 1810, Pipunculus Latreille, 1802 and Tomosvaryella Aczél, 1939. Zootaxa, 1672: 1-42.

Kehlmaier, C. \& Assmann, T. 2008. The European species of Chalarus Walker, 1834 revisited (Diptera: Pipunculidae). Zootaxa, 1936: 1-39.

Kehlmaier, M., Gibbs, D.J., \& Withers, P. 2019. New records of big-headed flies (Diptera: Pipunculidae) from the Mediterranean Basin. Bonn Zoological Bulletin, 68(1): 31-60. https:// doi.org/10.20363/BZB-2019.68.1.031
Kozánek, M. 1981. Genus Pipunculus Latreille (Diptera, Pipunculidae) in Czechoslovakia. Annotationes Zoologicae et Botanicae, 142: 1-16.

Motamedinia, B., Skevington, J.H., \& Kelso, S. 2020. Taxonomic revision of Dasydorylas Skevington, 2001 (Diptera, Pipunculidae) in the Middle East. PeerJ, 8:e8511. https://doi. org/10.7717/peerj.8511

Skevington, J.H. 2002. Revision of Australian Eudorylas Aczél (Diptera: Pipunculidae). Studia Dipterologica, 9: 621-672.

\section{Zoobank registrations}

\section{Present article:}

urn:1sid:zoobank.org:pub:C2E6FE04-32A3-437C-B7A5A81FA55EE5A5

Pipunculus adami Kozánek, sp. nov.: urn:1sid:zoobank.org:act:860FCCCC-F2A9-4FD6-A5D502FB1DE39FCA

\section{Pipunculus anatolicus Kozánek, sp. nov.:}

urn:1sid:zoobank.org:act:541D0E36-F16E-4C41-8EF159A512037D08

Pipunculus lindae Kozánek, sp. nov.: urn:1sid:zoobank.org:act:4B552904-CF2A-43BE-B77C809F2C5FCD3D 